\title{
Celiac Disease and Eosinophilic Esophagitis in Children
}

\author{
Madalina Adriana Bordea ${ }^{1, *}$, Gabriel Samasca, ${ }^{1,2}$, Nicolae Miü ${ }^{3}$ \\ ${ }^{1}$ Pediatrics Clinics II. Emergency Hospital for Children, Cluj-Napoca, Romania \\ ${ }^{2}$ Department of Immunology, Iuliu Hatieganu University of Medicine and Pharmacy, Cluj-Napoca, Romania \\ ${ }^{3}$ Department of Pediatrics II, Iuliu Hatieganu University of Medicine and Pharmacy, Cluj-Napoca, Romania \\ *Corresponding author: bordea_madalina@yahoo.com
}

Received September 10, 2014; Revised September 17, 2014; Accepted September 19, 2014

\begin{abstract}
Celiac disease is a chronic, $\mathrm{Th}_{1}$ - type immune-mediated disorder, characterized by small intestinal inflammation and villous atrophy, which leads to malabsorption of nutrients,after the ingestion of gluten or related proteins from rye and barley by genetically susceptible individuals. Exclusion of offending food antigens results in disease remission and reexposure leads to recurrence. Extraintestinal manifestations have been associated with celiac disease in children and adults. Eosinophilic esophagitis is a chronic, Th2- type immune-mediated disorder, isolated to the esophagus, which is most often triggered by exposure to food antigens in children and aeroallergens in adults. During the last decade, the increasing prevalence of celiac disease and eosinophilic esophagitis has been recognized in pediatric and adult populations all over the world. Case series have suggested an association between eosinophilic esophagitis and celiac disease. The prevalence of eosinophilic esophagitis in subjects with celiac disease was about 10-times that of the general population. The aim of this review is to analyse the similarity between these two frequent associated conditions. Coexistent eosinophilic esophagitis should be considered in children with celiac disease who have persistent esophageal symptoms.
\end{abstract}

Keywords: celiac disease, eosinophilic esophagitis, association, similarity

Cite This Article: Madalina Adriana Bordea, Gabriel Samasca, and Nicolae Miu, "Celiac Disease and Eosinophilic Esophagitis in Children.” International Journal of Celiac Disease, vol. 2, no. 3 (2014): 97-99. doi: 10.12691/ijcd-2-3-10.

\section{Epidemiology and Pathogeny}

Celiac disease is a chronic, Th1- type immune-mediated disorder, characterized by small intestinal inflammation and villous atrophy, which leads to malabsorption of nutrients, after the ingestion of gluten or related proteins from rye and barley by genetically susceptible individuals (expressing the human leukocyte antigen (HLA) class II molecules DQ2 or DQ8). Exclusion of offending food antigens (gluten or related proteins from rye and barley) results in disease remission and reexposure leads to recurrence [1]. Increased intestinal mucosal permeability secondary to celiac disease has been suggested as a contributing factor in the development of atopy. Damaged intestinal barrier may expose the local intestinal immune system to macromolecules and lead to transport of these undigested proteins to other body sites, facilitating development of hypersensitivity reactions in a predisposed individual in and away from the gastrointestinal [2]. Extraintestinal manifestations like : anemia, osteopenia, neurological disorders, menstrual abnormalities, infertility, failure to thrive, dermatitis herpetiformis, aphthous stomatitis, have been associated with celiac disease in children and adults [3].

Eosinophilic esophagitis is a chronic, immune mediated disorder, Th2-type allergic inflammation, isolated to the esophagus [4].
The increasing prevalence of eosinophilic esophagitis has been correlated with the increasing prevalence of atopy also. Eosinophilic esophagitis seems to be primarily a food antigen-driven disease in children, whereas in adults, mainly aeroallergen sensitization has been observed. Because the number and nature of dietary triggers greatly vary between individuals, no "one-size-fits-all" diet has been devised that can at once eliminate the offending antigens while ensuring complete nutrition for all ages as we do on celiac syndrome [5]. Replacement of the diet by aminoacid-based formula accomplishes the task, but cost, prolonged process for antigens reintroduction represent a great challenge for the patients. One alternative to the elemental diet is the 6-food elimination diet (SFED): milk, egg, soy, wheat, nuts and fish/shellfish. The advantage of SFED is the retention of a substantial portion of the diet. Which of the antigens is the trigger of inflammation is then determined by individual reintroduction followed by endoscopic biopsies.

Experimental evidence suggests that eosinophils play an important pathogenic role. Data published [6] showed that children with eosinophils over 15 per high power field were identified as having eosinophilic esophagitis. A subset of patients with eosinophilic esophagitis responds to acid suppressive therapy, indicating some overlap between eosinophilic esophagitis and gastroesophageal reflux disease. This is now considered as a separate entity named "proton pump inhibitors (PPI) responsive oesophageal eosinophilia” [7]. 
Surface activation markers (CD66b) and intracellular phosphoepitopes (phosphorylated forms of signal transducer and activator of transcription 1 or 6 ) are both significantly higher in peripheral eosinophils. Detection of these marker levels in the blood may be beneficial for diagnose eosinophilic esophagitis in the near future. Regulation of eosinophil maturation, recruitment and survival is under the control of interleukin 5 [8].

There is a male predominance in eosinophilic esophagitis, while female predominance is specific for celiac disease [9].

The prevalence of eosinophilic esophagitis in subjects with celiac disease was about 10-times that of the general population. There are many original articles in witch association between celiac disease and eosinophilic esophagitis has been reported $[5,10]$.

As a conclusion, both conditions (celiac disease and eosinophilic esophagitis) are caused by aberrant, but distinct, immune responses to ingested different antigens and can be responsive to food elimination diets.

\section{Clinical Presentation and Histologic Feature}

Vomiting and failure to thrive are seen in children, while dysphagia, heartburn and food impaction with spontaneous perforation of the esophagus (Boerhaave's syndrome) are seen in adults with eosinophilic esophagitis. They also may have: globus, irritability, anorexia and water brash.

Celiac disease has many symptoms also. According to some experts, there are about 300 possible symptoms of the disease. Different people will experience the disease in different ways because the symptoms vary greatly from one person to another [5].

Common symptoms for infants and children are: failure to thrive, vomiting, chronic diarrhea (bloody diarrhea), chronic constipation, abdominal pain, fatigue or irritability.

In contrast, adults have: anemia, osteopenia, neurological disorders, menstrual abnormalities, infertility, dermatitis herpetiformis, aphthous stomatitis and only few digestive problems [5].

Because the symptoms greatly vary between individuals, eosinophilic esophagitis and celiac disease diagnosis can be frequent challenging.

The major pathologic features of eosinophilic esophagitis include eosinophilic micro abscesses, surface layering of eosinophils often associated with surface sloughing of necrotic squamous cells and peak eosinophil counts greater than 15 per high power field within the squamous epithelium.

The proximal small intestine is the major site of celiac disease. Increased intraepithelial lymphocytosis, with or without concomitant villous atrophy, is the characteristic histologic finding. While histologic examination remains the "gold standard" for diagnosis of celiac disease, changes can be subtle when duodenal villous architecture is intact, and villous atrophy may also be encountered in various other conditions [2,5].

In both condition, histologic examination represent the gold standard for diagnosis. The histologic features are different from one to another but, in the same time, are both non-specific.

\section{Diagnostic Test and Criteria}

The combination of IgE blood level, skin prick testing and patch testing can identify potential causative foods that might contribute to the pathogenesis of eosinophilic esophagitis, but the predecitive values are low. Because symptoms do not necessarily return in the early phase of antigen reintroduction, endoscopic biopsy has been performed to ascertain the impact of the antigen on the mucosa [11].

Similarly, serologic testing using an enzyme-linked immunosorbent assay for antitissuetransglutaminase (tTG) antibodies, which recognizes an important enzyme in celiac disease pathogenesis, is not fully sensitive or specific for the disease. In practice, a combination of clinical suspicion, morphologic abnormality, and positive serologic findings is used for the initial diagnosis of most patients with celiac disease [5].

Three criteria must be met to diagnose eosinophilic esophagitis: clinical symptoms of esophageal dysfunction, an esophageal biopsy with a peak eosinophil count of at least 15 eosinophils per high-power microscopy field and exclusion of other possible causes of esophageal eosinophilia [12].

Initial celiac disease evaluation is based on a combination of positive celiac disease -specific serological tests, histological findings in the intestinal biopsy, celiac disease-predisposing gene encoding HLA DQ2 or DQ8, family and medical history of celiac disease, and clinical or histological response to gluten free diet. However, celiac disease diagnosis can be challenging in some nonresponsive patients to gluten free diet [10].

\section{Conclusions}

Celiac disease and eosinophilic esophagitis are distinct clinical conditions, except for a few minor similarities like presence of atopy, many ways of clinical presentation and serological tests or histologic features, both non-specific. These similarities are common characteristics of all immune-mediated disorders,infact. Celiac disease is a Th1 mediated disorder, triggered by the ingestion of gluten and affects females:males at a ratio of $2: 1$. By contrast, eosinophilic esophagitis has been shown to be a Th2mediated disorder, isolated to the esophagus, which is triggered by exposure to dietary allergens, characterized by inflammation of the esophageal mucosa by $\mathrm{T}$ lymphocytes, mast cells and eosinophils and predominates in males, with a 3:1 ratio to females.

Although there are fundamental differences in the pathophysiological mechanisms involved in eosinophilic esophagitis and celiac disease, these conditions may coexist and the prevalence is higher in children and adults. Coexistent eosinophilic esophagitis should be considered in children with celiac disease who have persistent esophageal symptoms.

Celiac disease and eosinophilic esophagitis are distinct disorders with specific clinico-pathological characteristics.

In light of many recent reports, celiac disease and eosinophilic esophagitis is a true association.

\section{References}


[1] Leslie C, Mews C, Charles A, Ravikumara M. Celiac disease and eosinophilic esophagitis: a true association. J Pediatr Gastroenterol Nutr 2010; 50: 397-9.

[2] Stewart MJ, Shaffer E, Urbanski SJ, Beck PL, Storr MA. The association between celiac disease and eosinophilic esophagitis in children and adults. BMC Gastroenterol 2013; 13: 11.

[3] Ludvigsson JF, Aro P, Walker MM, Vieth M, Agréus L, Talley NJ, Murray JA, Ronkainen J. Celiac disease, eosinophilic esophagitis and gastroesophageal reflux disease, an adult population-based study. Scand J Gastroenterol 2013; 48: 808-14.

[4] Thompson JS, Lebwohl B, Reilly NR, Talley NJ, Bhagat G, Green $\mathrm{PH}$. Increased incidence of eosinophilic esophagitis in children and adults with celiac disease. J ClinGastroenterol 2012; 46: e6e11.

[5] Pellicano R, De Angelis C, Ribaldone DG, Fagoonee S, Astegiano M. 2013 update on celiac disease and eosinophilicesophagitis. Gluten-free diet does not appear to induce endoscopic remission of eosinophilic esophagitis in children with coexistent celiac disease. Nutrients 2013; 5: 3329-36.

[6] Sánchez-García S, Ibáñez MD, Martinez-Gómez MJ, Escudero C, Vereda A, Fernández-Rodríguez M, Rodríguez del Río P. Eosinophilic esophagitis, celiac disease, and immunoglobulin E- mediated allergy in a 2-year-old child. J Investig Allergol Clin Immunol 2011; 21: 73-5.

[7] Soon IS, Butzner JD, Kaplan GG, deBruyn JC. Incidence and prevalence of eosinophilic esophagitis in children. J Pediatr Gastroenterol Nutr 2013; 57: 72-80.

[8] Atkins D, Furuta GT. Mucosal immunology, eosinophilic esophagitis, and other intestinal inflammatory diseases. J Pediatr Gastroenterol Nutr 2013; 57: 72-80.

[9] Lucendo AJ, Arias Á, Pérez-Martínez I, López-Vázquez A, Ontañón-Rodríguez J, González-Castillo S, De Rezende LC, Rodrigo L. Adult patients with eosinophilic esophagitis do not show an increased frequency of the HLA-DQ2/DQ8 genotypes predisposing to celiac disease. Dig Dis Sci 2011; 56: 1107-11.

[10] Lucendo AJ. Esophageal manifestations of celiac disease. Dis Esophagus 2011; 24: 470-5.

[11] Ho MH, Wong WH, Chang C. Clinical spectrum of food allergies: a comprehensive review. Clin Rev Allergy Immunol 2014; 46: 225-40.

[12] Bordea MA, Mosteanu O, Pop TA, Gheban D, Samasca G, Miu N. Eosinophilic esophagitis. Acta Gastro-Enterologica Belgica 2013; 76: 407-412. 